\title{
Existence of solutions for fractional difference equations via topological degree methods
}

\author{
Jia Wei He ${ }^{1}$, Lu Zhang1, Yong Zhou ${ }^{1,2^{*}}$ (1) and Bashir Ahmad ${ }^{2}$
}

\section{${ }^{*}$ Correspondence:}

yzhou@xtu.edu.cn

${ }^{1}$ Faculty of Mathematics and

Computational Science, Xiangtan

University, Hunan, P.R. China

${ }^{2}$ Nonlinear Analysis and Applied

Mathematics (NAAM) Research

Group, Faculty of Science, King

Abdulaziz University, Jeddah, Saudi

Arabia

\begin{abstract}
In this paper, a class of nonlinear fractional difference equations with Caputo-like difference operator is considered. Some existence results for the given equation are obtained by means of topological degree methods. Three examples are constructed for the illustration of the obtained theory.
\end{abstract}

MSC: 34D20; 39A06; 39A13

Keywords: Fractional differences equations; Caputo-like difference operator; Existence; Topological degree methods

\section{Introduction}

In this paper, we consider the following nonlinear fractional difference equations:

$$
\left\{\begin{array}{l}
{ }^{C} \Delta^{\alpha} u(t)=f(t+\alpha, u(t+\alpha)), \quad t \in \mathbb{N}_{1-\alpha}, 0<\alpha<1, \\
u(0)=u_{0}
\end{array}\right.
$$

where ${ }^{C} \Delta^{\alpha}$ is a Caputo-like fractional difference operator, $f:[0, \infty) \times \mathbb{R} \rightarrow \mathbb{R}$ is a given continuous function and $\mathbb{N}_{1-\alpha}=\{1-\alpha, 2-\alpha, \ldots\}$.

Fractional differential operators appear naturally in modeling many phenomena in various fields of engineering, physics and economics, for example, nonlinear oscillations of earthquakes, seepage flow in porous media and dynamic traffic flow model. For details, we refer the reader to the monographs by Kilbas et al. [1], Diethelm [2], Zhou [3, 4], and to [5-12]. The theory of fractional difference equations has been rapidly developed in recent years (see [13-17]). It can elegantly describe certain behaviors in discrete fractional calculus or generalized difference equations. There are several definitions of fractional sum/difference operators proposed by many mathematicians such as Gray and Zhang [18], Atici and Eloe [19], Abdeljawad [20]. Goodrich [21] studied existence of positive solutions for fractional difference equations with initial-boundary data, Chen et al. [22-24] obtained some asymptotic stability results for some nonlinear fractional difference equations. However, to the best of our knowledge, fractional difference equations have not yet been investigated with the aid of topological degree methods.

In this paper, we show existence of solutions for nonlinear fractional difference equations by applying a fixed point theorem due to Isaia [25], which was obtained via coinci-

(c) The Author(s) 2018. This article is distributed under the terms of the Creative Commons Attribution 4.0 International License (http://creativecommons.org/licenses/by/4.0/), which permits unrestricted use, distribution, and reproduction in any medium, provided you give appropriate credit to the original author(s) and the source, provide a link to the Creative Commons license, and indicate if changes were made. 
dence degree theory for condensing maps. The rest of the article is organized as follows. In Sect. 2, we introduce some important notions about fractional difference operators and topological degree theory, while Sect. 3 contains our main existence results for Eq. (1.1).

\section{Preliminaries}

For real numbers $a$ and for $\alpha \in(0,1)$, we denote $\mathbb{N}_{a}=\{a, a+1, \ldots\}, \mathbb{N}_{a+\alpha}=\{a+\alpha$, $a+\alpha+1, \ldots\}$ and $\mathbb{N}_{1}=\mathbb{N}$. The forward Euler difference operator $\Delta$ is defined by $\Delta u(t):=$ $u(t+1)-u(t), t \in \mathbb{N}_{a}$.

Definition 2.1 ([19]) Let $\alpha>0$ be given. The fractional sum of order $\alpha$ is defined as

$$
\Delta^{-\alpha} u(t)=\frac{1}{\Gamma(\alpha)} \sum_{s=a}^{t-\alpha}(t-s-1)^{(\alpha-1)} u(s)
$$

where $u$ is given for $s=a \bmod (1), \Delta^{-\alpha} u(t)$ is defined for $t=(a+\alpha) \bmod (1)$, and the falling factorial function is

$$
t^{(\alpha)}=\frac{\Gamma(t+1)}{\Gamma(t+1-\alpha)} .
$$

The fractional sum $\Delta^{-\alpha}$ maps functions defined on $\mathbb{N}_{a}$ to the functions defined on $\mathbb{N}_{a+\alpha}$.

Definition 2.2 Let $\mu>0$ be such that $m-1<\mu<m$, where $m$ denotes a positive integer and $m=\lceil\mu\rceil,\lceil\cdot\rceil$ ceiling of number. Set $v=m-\mu$. The Caputo-like fractional difference operator of order $\alpha>0$ is defined by

$$
{ }^{C} \Delta^{\mu} u(t):=\Delta^{-v}\left(\Delta^{m} u(t)\right)=\frac{1}{\Gamma(\mu)} \sum_{s=a}^{t-v}(t-s-1)^{(v-1)} \Delta^{m} u(s), \quad t \in \mathbb{N}_{a+v},
$$

where $\Delta^{m}$ is the $m$ th order forward difference operator. The fractional Caputo-like difference operator ${ }^{C} \Delta^{\mu}$ maps functions defined on $\mathbb{N}_{a}$ to the functions defined on $\mathbb{N}_{a+m-\mu}$.

Theorem 2.1 Let $u$ be a real value function defined on $\mathbb{N}_{a}$ and $\mu, v>0$. Then

$$
\Delta^{-\mu}\left(\Delta^{-v} u(t)\right)=\Delta^{-(\mu+v)} u(t)=\Delta^{-v}\left(\Delta^{-\mu} u(t)\right) .
$$

Lemma 2.1 ([24]) Let $0<\mu<1$. Then

$$
u(t)=u(0)+\frac{1}{\Gamma(\mu)} \sum_{s=1-\mu}^{t-\mu}(t-s-1)^{(\mu-1) C} \Delta^{\mu} u(s), \quad \text { for } t \in \mathbb{N}
$$

where $u$ is defined on $\mathbb{N}_{0}$ and ${ }^{C} \Delta^{\mu}$ is defined on $\mathbb{N}_{1-\mu}$.

Lemma 2.2 ([13]) Assume that $\mu+v+1$ is not a nonpositive integer with $\mu \neq-1$. Then

$$
\Delta^{-v} t^{(\mu)}=\frac{\Gamma(\mu+1)}{\Gamma(\mu+v+1)} t^{(\mu+v)}
$$


Definition 2.3 Let $\Omega \subset X$ and $F: \Omega \rightarrow X$ be a continuous bounded map. We say that $F$ is $\sigma$-Lipschitz if there exists $\kappa \geq 0$ such that

$$
\sigma(F(B)) \leq \kappa \sigma(B), \quad \text { for any bounded } B \subset \Omega
$$

In case $\kappa<1$, we call $F$ a strict $\sigma$-contraction. We say that $F$ is $\sigma$-condensing if $\sigma(F(B))<$ $\sigma(B)$ for any bounded $B \subset \Omega$ with $\sigma(B)>0$. In other words, $\sigma(F(B)) \geq \sigma(B)$ implies $\sigma(B)=0$. The aforementioned $\sigma$ is the Kuratowski measure of noncompactness.

Proposition 2.1 If $F: \Omega \rightarrow X$ is compact, then $F$ is $\sigma$-Lipschitz with constant $\kappa=0$.

Let

$$
\mathfrak{T}=\left\{(I-F, \Omega, y): \Omega \subset X \text { open and bounded, } F \in C_{\sigma}(\bar{\Omega}), y \in X \backslash(I-F)(\partial \Omega)\right\}
$$

be the family of the admissible triplets, where $C_{\sigma}(\bar{\Omega})$ is defined by the class of all $\sigma$ condensing maps $F: \bar{\Omega} \rightarrow X$.

Theorem 2.2([25]) Let $F: X \rightarrow X$ be $\sigma$-condensing and

$$
\mathfrak{T}=\{x \in X: \text { there exists } \lambda \in[0,1] \text { such that } x=\lambda F x\} .
$$

If $\mathfrak{T}$ is a bounded set in $X$, then there exists $r>0$ such that $\mathfrak{T} \subset B_{r}(0)$, and for a degree function $D: \mathfrak{T} \rightarrow \mathbb{Z}$,

$$
D\left(I-\lambda F, B_{r}(0), 0\right)=1, \quad \text { for any } \lambda \in[0,1] .
$$

Then $F$ has at least one fixed point and the set of the fixed points of $F$ lies in $B_{r}(0)$.

The space $l_{n_{0}}^{\infty}$ is the set of real sequences defined on the set of positive integers where any individual sequence is bounded with respect to the usual supremum norm. It is well known that $l_{n_{0}}^{\infty}$ is a Banach space under the supremum norm.

Definition 2.4 A set $\Omega$ of sequences in $l_{n_{0}}^{\infty}$ is uniformly Cauchy (or equi-Cauchy) if, for every $\varepsilon>0$, there exists an integer $N$ such that $|x(i)-x(j)|<\varepsilon$, whenever $i, j>N$ for any $x=\{x(n)\}$ in $\Omega$.

Theorem 2.3 (Discrete Arzela-Ascoli's theorem) A bounded uniformly Cauchy subset $\Omega$ of $l_{n_{0}}^{\infty}$ is relatively compact.

Lemma 2.3 ([23]) Assume that $\beta>1$ and $\gamma>0$. Then

$$
\left(t^{(-\gamma)}\right)^{\beta} \leq \frac{\Gamma(1+\beta \gamma)}{\Gamma^{\beta}(1+\gamma)} t^{(-\beta \gamma)}, \quad \text { for } t \in \mathbb{N} .
$$

Lemma 2.4 ([19]) Assume that the falling factorial functions are well defined, then

(i) If $0<\beta<1$ and $\gamma>0$, then $\left(t^{(-\gamma)}\right)^{\beta} \leq t^{(-\beta \gamma)}$;

(ii) $t^{(\alpha+\beta)}=(t-\beta)^{(\alpha)} t^{(\beta)}$. 
Remark 2.1 Obviously, If $\beta=1$ in Lemma 2.3 and $\beta=1$ in Lemma 2.4(i), then the resulting expressions still hold true.

Lemma 2.5 Assume that the falling factorial functions are well defined. Then

$$
t^{(\beta)} \cdot t^{(-\alpha)} \leq t^{(\beta-\alpha)}, \quad \text { for } \alpha \geq 0, \beta \geq 0, t>\beta-1 \text {. }
$$

Proof From the definition of falling factorial function $t^{(\cdot)}$, we deduce that $t^{(-\alpha)}$ is nonincreasing for any $\alpha \geq 0, t>-1$, that is, $t^{(-\alpha)} \leq(t-\beta)^{(-\alpha)}$ for $\alpha \geq 0, \beta \geq 0$ and $t>\beta-1$. Indeed, it holds true obviously for $\alpha=0$ or $\beta=0$. Then, for $\alpha>0, \beta>0$ and $t>\beta-1$, we find that

$$
\begin{aligned}
\frac{(t-\beta)^{(-\alpha)}}{t^{(-\alpha)}} & =\frac{\Gamma(t+1-\beta)}{\Gamma(t+1-\beta+\alpha)} \frac{\Gamma(t+1+\alpha)}{\Gamma(t+1)} \\
& =\frac{\Gamma(t-\beta+1) \Gamma(t+\alpha+1)}{\Gamma(\lambda(t+\alpha+1)+(1-\lambda)(t-\beta+1)) \Gamma((1-\lambda)(t+\alpha+1)+\lambda(t-\beta+1))},
\end{aligned}
$$

where $\lambda=\frac{\alpha}{\beta+\alpha} \in(0,1)$. Then, following the log-convexity property of the gamma function, we have

$$
\begin{aligned}
\frac{(t-\beta)^{(-\alpha)}}{t^{(-\alpha)}} & =\frac{\Gamma(t-\beta+1) \Gamma(t+\alpha+1)}{\Gamma(\lambda(t+\alpha+1)+(1-\lambda)(t-\beta+1)) \Gamma((1-\lambda)(t+\alpha+1)+\lambda(t-\beta+1))} \\
& \geq \frac{\Gamma(t-\beta+1) \Gamma(t+\alpha+1)}{(\Gamma(t+\alpha+1))^{\lambda+1-\lambda}(\Gamma(t-\beta+1))^{1-\lambda+\lambda}}=1 .
\end{aligned}
$$

Therefore, $t^{(-\alpha)} \leq(t-\beta)^{(-\alpha)}$ for $\alpha>0, \beta>0$ and $t>\beta-1$. By Lemma 2.4(ii), we obtain

$$
t^{(-\alpha)} \cdot t^{(\beta)} \leq(t-\beta)^{(-\alpha)} \cdot t^{(\beta)}=t^{(\beta-\alpha)}
$$

\section{Main results}

In this section, we study the existence and uniqueness of solutions for nonlinear fractional difference equations.

Let $X:=l_{1}^{\infty}$ be the set of all real sequences $\{x(t)\}_{t=1}^{\infty}$ with norm

$$
\|x\|=\sup _{t \in \mathbb{N}}|x(t)|
$$

Then $X$ is a Banach space.

Lemma 3.1 Letf be a real-valued function. Then the problem (1.1) has one solution if and only if $u$ is a solution of the following fractional Taylor difference equations:

$$
u(t)= \begin{cases}u_{0}, & t=0 \\ u_{0}+\frac{1}{\Gamma(\alpha)} \sum_{s=1-\alpha}^{t-\alpha}(t-s-1)^{(\alpha-1)} f(s+\alpha, u(s+\alpha)), & t \in \mathbb{N}\end{cases}
$$

where $0<\alpha<1$.

Proof The proof is similar to that of [24, Lemma 2.4]. So we omit it. 
Now, for $\gamma>0$, we define

$$
S=\left\{x \in X:\left|x(t)-x_{0}\right| \leq t^{(-\gamma)}, x(0)=x_{0}, \text { for } t \in \mathbb{N}\right\}
$$

Clearly the set $S$ consists of nonempty bounded and closed subsets of $X$.

For any $u \in S$, define an operator $P: X \rightarrow X$ as follows:

$$
(P u)(t)= \begin{cases}u_{0}, & t=0 \\ u_{0}+\frac{1}{\Gamma(\alpha)} \sum_{s=1-\alpha}^{t-\alpha}(t-s-1)^{(\alpha-1)} f(s+\alpha, u(s+\alpha)), & t \in \mathbb{N}\end{cases}
$$

Observe that the existence of a solution $u$ for (1.1) is equivalent to that of a fixed point $u$ in $S$ such that $u=P u$ holds.

Lemma 3.2 Assume that

(H1) there exist $C_{f}>0, \beta \in(\alpha, 1)$, and $\gamma=\frac{1}{2}(\beta-\alpha)$ such that

$$
\frac{C_{f} \Gamma(1-\beta)}{\Gamma(1+\alpha-\beta)} \leq 1
$$

Iff satisfies

$$
|f(t, u)| \leq C_{f} t^{(-\beta)}
$$

then the operator $P$ is continuous and $P$ maps $S$ into $S$.

Proof Let $u \in S$. Since $0<\alpha<\beta$, by the non-increasing characteristic of $t^{(-\alpha)}$ for any $t \in \mathbb{N}$, it follows by Lemma 2.5 together with a given $\varepsilon>0$ that there exists $n_{1} \in \mathbb{N}$, such that, for $n \in \mathbb{N}_{n_{1}}$, we have

$$
\frac{C_{f} \Gamma(1-\beta)}{\Gamma(1+\alpha-\beta)} \frac{\Gamma(n+\alpha+1)}{\Gamma(n+\beta+1)}<\frac{\varepsilon}{2} .
$$

By the definition of $P$, Lemma 2.2, Lemma 2.4(ii), (H1), and inequality (3.4), for $t \in \mathbb{N}$, we find that

$$
\begin{aligned}
\left|(P u)(t)-u_{0}\right| & =\frac{1}{\Gamma(\alpha)} \sum_{s=1-\alpha}^{t-\alpha}(t-s-1)^{(\alpha-1)}|f(s+\alpha, u(s+\alpha))| \\
& \leq \frac{C_{f}}{\Gamma(\alpha)} \sum_{s=1-\alpha}^{t-\alpha}(t-s-1)^{(\alpha-1)}(s+\alpha)^{(-\beta)} \\
& =C_{f} \Delta^{-\alpha}(t+\alpha)^{(-\beta)} \\
& =\frac{C_{f} \Gamma(1-\beta)}{\Gamma(1+\alpha-\beta)}(t+\alpha)^{(\alpha-\beta)} \\
& =\frac{C_{f} \Gamma(1-\beta)}{\Gamma(1+\alpha-\beta)}(t+\alpha+\gamma)^{(\gamma+\alpha-\beta)}(t+\alpha)^{(-\gamma)} \\
& \leq \frac{C_{f} \Gamma(1-\beta)}{\Gamma(1+\alpha-\beta)} t^{(-\gamma)} t^{(-\gamma)}
\end{aligned}
$$




$$
\begin{aligned}
& \leq \frac{C_{f} \Gamma(1-\beta)}{\Gamma(1+\alpha-\beta)} \frac{1}{\Gamma(2+\gamma)} t^{(-\gamma)} \\
& \leq t^{(-\gamma)}
\end{aligned}
$$

where we applied the inequality $1 \leq \Gamma(\theta)$ for $\theta \geq 2$. Hence $P S \subset S$.

Let $\left\{u_{m}\right\}_{m=1}^{\infty}$ be a sequence of $S$ such that $u_{m} \rightarrow u$ as $m \rightarrow \infty$. Then, for $t \in \mathbb{N}_{n_{1}}$, by (H1) and (3.5), we obtain

$$
\begin{aligned}
\left|\left(P u_{m}\right)(t)-(P u)(t)\right| & \left|\frac{1}{\Gamma(\alpha)} \sum_{s=1-\alpha}^{t-\alpha}(t-s-1)^{(\alpha-1)}\left(f\left(s+\alpha, u_{m}(s+\alpha)\right)-f(s+\alpha, u(s+\alpha))\right)\right| \\
\leq & \frac{1}{\Gamma(\alpha)} \sum_{s=1-\alpha}^{t-\alpha}(t-s-1)^{(\alpha-1)}\left|f\left(s+\alpha, u_{m}(s+\alpha)\right)\right| \\
& \quad+\frac{1}{\Gamma(\alpha)} \sum_{s=1-\alpha}^{t-\alpha}(t-s-1)^{(\alpha-1)}|f(s+\alpha, u(s+\alpha))| \\
\leq & 2 C_{f} \frac{1}{\Gamma(\alpha)} \sum_{s=1-\alpha}^{t-\alpha}(t-s-1)^{(\alpha-1)}(s+\alpha)^{(-\beta)} \\
= & \frac{2 C_{f} \Gamma(1-\beta)}{\Gamma(1+\alpha-\beta)}(t+\alpha)^{(\alpha-\beta)} \\
\leq & \frac{2 C_{f} \Gamma(1-\beta)}{\Gamma(1+\alpha-\beta)}\left(n_{1}+\alpha\right)^{(\alpha-\beta)}<\varepsilon .
\end{aligned}
$$

For $t \in\left\{1, \ldots, n_{1}-1\right\}$, in view of the continuity of $f$, we get

$$
\begin{aligned}
& \left|\left(P u_{m}\right)(t)-(P u)(t)\right| \\
& \quad \leq \frac{1}{\Gamma(\alpha)} \sum_{s=1-\alpha}^{t-\alpha}(t-s-1)^{(\alpha-1)}\left|f\left(s+\alpha, u_{m}(s+\alpha)\right)-f(s+\alpha, u(s+\alpha))\right| \\
& \quad \leq \frac{1}{\Gamma(\alpha)} \sum_{s=1-\alpha}^{t-\alpha}(t-s-1)^{(\alpha-1)} \max _{s \in\left[1-\alpha, \ldots, n_{1}-1-\alpha\right]}\left|f\left(s+\alpha, u_{m}(s+\alpha)\right)-f(s+\alpha, u(s+\alpha))\right| \\
& \quad=\frac{t^{(\alpha)}}{\Gamma(\alpha+1)} \max _{s \in\left[1-\alpha, \ldots, n_{1}-1-\alpha\right]}\left|f\left(s+\alpha, u_{m}(s+\alpha)\right)-f(s+\alpha, u(s+\alpha))\right| \\
& \quad \leq \frac{\left(n_{1}-1\right)^{(\alpha)}}{\Gamma(\alpha+1)} \max _{s \in\left[1-\alpha, \ldots, n_{1}-1-\alpha\right]}\left|f\left(s+\alpha, u_{m}(s+\alpha)\right)-f(s+\alpha, u(s+\alpha))\right| \\
& \quad \rightarrow 0, \quad \text { as } m \rightarrow \infty .
\end{aligned}
$$

Thus, for $t \in \mathbb{N}$, it is clear that

$$
\left|\left(P u_{m}\right)(t)-(P u)(t)\right| \rightarrow 0, \quad \text { as } m \rightarrow \infty .
$$

Hence $P$ is continuous on $S$.

Lemma 3.3 Assume that (H1) holds. Then PS is a compact subset of X. 
Proof From Lemma 3.2, we know that $P S$ is a bounded subset of $X$. Next, we will show that $P$ is compact.

Let $t_{1}, t_{2} \in \mathbb{N}_{n_{1}}$ with $t_{2}>t_{1}$. From (3.5), we have

$$
\begin{aligned}
&\left|(P u)\left(t_{2}\right)-(P u)\left(t_{1}\right)\right| \leq \frac{1}{\Gamma(\alpha)} \sum_{s=1-\alpha}^{t_{2}-\alpha}\left(t_{2}-s-1\right)^{(\alpha-1)}|f(s+\alpha, u(s+\alpha))| \\
&+\frac{1}{\Gamma(\alpha)} \sum_{s=1-\alpha}^{t_{1}-\alpha}\left(t_{1}-s-1\right)^{(\alpha-1)}|f(s+\alpha, u(s+\alpha))| \\
& \leq \frac{C_{f} \Gamma(1-\beta)}{\Gamma(1+\alpha-\beta)}\left(t_{2}+\alpha\right)^{(\alpha-\beta)}+\frac{C_{f} \Gamma(1-\beta)}{\Gamma(1+\alpha-\beta)}\left(t_{1}+\alpha\right)^{(\alpha-\beta)} \\
&<\varepsilon .
\end{aligned}
$$

Hence, for an arbitrary choice of $\varepsilon,\{P u: u \in S\}$ is a uniformly Cauchy subset of $X$ by Definition 2.4. From Lemma 3.2, we know that $\{P u: u \in S\}$ is bounded. Thus a direct application Theorem 2.3 implies that $P S$ is relatively compact.

Theorem 3.1 Assume that (H1) holds. Then the problem (1.1) has at least one solution $u \in S$ and the set of solutions of (1.1) is bounded in $S$.

Proof Let $P: S \rightarrow S$ be the operator defined by (3.3). We know that $P$ is continuous and bounded by Lemma 3.2. Moreover, by Lemma 3.3, $P$ is compact. Hence, it follows by Proposition 2.1 that $P$ is a strict $\sigma$-contraction with constant zero.

Let us set

$$
S_{0}=\{u \in S: \text { there exists } \lambda \in[0,1] \text { such that } u=\lambda P u\}
$$

and show that $S_{0}$ is bounded in $S$. Consider $u \in S_{0}$ and $\lambda \in[0,1]$ such that $u=\lambda P u$. Using (3.6), we find that

$$
\|u\|=\lambda\|P u\| \leq\left\|u_{0}\right\|+\frac{1}{\Gamma(2+\gamma)}, \quad \text { for } t \in \mathbb{N},
$$

which implies that $S_{0}$ is bounded in $S$. If not, we suppose by contradiction, $\rho:=\|u\| \rightarrow \infty$. Dividing both sides of (3.7) by $\rho$, and taking the limit $\rho \rightarrow \infty$, we have

$$
1 \leq \lim _{\rho \rightarrow \infty} \frac{1}{\rho}\left(\left\|u_{0}\right\|+\frac{1}{\Gamma(2+\gamma)}\right)=0
$$

which is an obvious contradiction. Consequently, by Theorem 2.2, we deduce that $P$ has at least one solution $u^{*}$ in $S$.

Theorem 3.2 Assume that (H1) holds. Furthermore, suppose that

(H2) there exist $L>0$ and $\xi>\alpha$ such that

$$
|f(t, y)-f(t, x)| \leq L t^{(-\xi)}|x-y|, \quad \text { for any } x, y \in S, \text { for } t \in \mathbb{N}_{0} .
$$


Then the problem (1.1) has a unique solution provided that

$$
\frac{L \Gamma(1-\xi)}{\Gamma(1+\alpha-\xi)}<1
$$

Proof Let $u, v \in S$ be two solutions of (1.1). Then, for $t \in \mathbb{N}$, applying assumption (H2), we have

$$
\begin{aligned}
|u(t)-v(t)| & \leq \frac{1}{\Gamma(\alpha)} \sum_{s=1-\alpha}^{t-\alpha}(t-s-1)^{(\alpha-1)}|f(s+\alpha, u(s+\alpha))-f(s+\alpha, v(s+\alpha))| \\
& \leq \frac{L}{\Gamma(\alpha)} \sum_{s=1-\alpha}^{t-\alpha}(t-s-1)^{(\alpha-1)}(s+\alpha)^{(-\xi)}\|u-v\| \\
& =\frac{L \Gamma(1-\xi)}{\Gamma(1+\alpha-\xi)}(t+\alpha)^{(\alpha-\xi)}\|u-v\| \\
& \leq \frac{L \Gamma(1-\xi)}{\Gamma(1+\alpha-\xi)} t^{(\alpha-\xi)}\|u-v\| \\
& \leq \frac{L \Gamma(1-\xi)}{\Gamma(1+\alpha-\xi)}\|u-v\|
\end{aligned}
$$

which implies that $\|u-v\|=0$ by virtue of (3.9). Hence, there exists a unique solution of (1.1).

Example 3.1 Let us consider the following fractional difference equations:

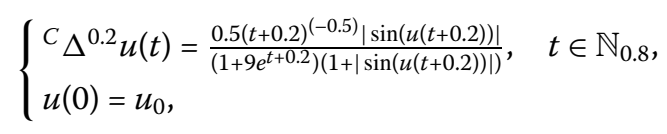

where $f(t, u)=\frac{0.5 t^{(-0.5)}|\sin (u(t))|}{\left(1+9 e^{t}\right)(1+|\sin (u(t))|)}$ for $t \in \mathbb{N}_{0}$.

Since

$$
|f(t, u)|=\left|\frac{0.5 t^{(-0.5)}|\sin (u(t))|}{\left(1+9 e^{t}\right)(1+|\sin (u(t))|)}\right| \leq 0.5 t^{(-0.5)}
$$

and, from the above given data, we find that

$$
\frac{0.5 \Gamma(0.5)}{\Gamma(0.7)} \approx 0.6827<1
$$

therefore, condition (H1) holds. Thus, by Theorem 3.1, there exists at least one solution in $S$. Furthermore,

$$
\begin{aligned}
|f(t, u)-f(t, v)| & =\left|\frac{0.5 t^{(-0.5)}|\sin (u(t))|}{\left(1+9 e^{t}\right)(1+|\sin (u(t))|)}-\frac{0.5 t^{(-0.5)}|\sin (v(t))|}{\left(1+9 e^{t}\right)(1+|\sin (v(t))|)}\right| \\
& \leq \frac{0.5 t^{(-0.5)}}{1+9 e^{t}}\left|\frac{|\sin (u(t))|}{1+|\sin (u(t))|}-\frac{|\sin (v(t))|}{1+|\sin (v(t))|}\right| \\
& \leq \frac{0.5 t^{(-0.5)}}{1+9 e^{t}} \frac{|| \sin (u(t))|-| \sin (v(t))||}{(1+|\sin (u(t))|)(1+|\sin (v(t))|)}
\end{aligned}
$$




$$
\begin{aligned}
& \leq \frac{0.5 t^{(-0.5)}}{1+9 e^{t}} \frac{|\sin (u(t))-\sin (v(t))|}{(1+|\sin (u(t))|)(1+|\sin (v(t))|)} \\
& \leq t^{(-0.5)}\left|\sin \left(\frac{u(t)-v(t)}{2}\right) \cos \left(\frac{u(t)+v(t)}{2}\right)\right| \\
& \leq 0.5 t^{(-0.5)}\|u-v\|,
\end{aligned}
$$

which implies that $(\mathrm{H} 2)$ holds. With the given data, we also find that the inequality (3.9) holds. Thus the problem (3.10) admits a unique solution.

Theorem 3.3 Let $\frac{1}{1-\alpha}<q$. Assume that

(H3) there exist $C_{f}^{\prime}>0, \eta \in(0, q(1-\alpha)-1)$ and $\gamma \in\left(\frac{\eta+\alpha}{q-1}, \frac{1+\eta}{q}\right)$ such that

$$
\frac{C_{f}^{\prime} \Gamma(1+\gamma q) \Gamma(1+\eta-\gamma q)}{\Gamma^{q}(1+\gamma) \Gamma(1+\alpha+\eta-\gamma q)} \leq 1
$$

Iff satisfies

$$
|f(t, u)| \leq C_{f}^{\prime} t^{(\eta)}|u(t)|^{q}
$$

then the problem (1.1) has at least one solution $u \in S$ and the set of solutions of (1.1) is bounded in $S$.

Proof From the definition of $P$, by the non-increasing characteristic of $t^{(-\alpha)}$ for any $t \in \mathbb{N}$, together with (H3), Lemma 2.3, Lemma 2.4(ii) and Lemma 2.5, for any $u \in S$, we have

$$
\begin{aligned}
\left|(P u)(t)-u_{0}\right| & \leq \frac{1}{\Gamma(\alpha)} \sum_{s=1-\alpha}^{t-\alpha}(t-s-1)^{(\alpha-1)}|f(s+\alpha, u(s+\alpha))| \\
& \leq \frac{C_{f}^{\prime}}{\Gamma(\alpha)} \sum_{s=1-\alpha}^{t-\alpha}(t-s-1)^{(\alpha-1)}(s+\alpha)^{(\eta)}|u(s+\alpha)|^{q} \\
& \leq \frac{C_{f}^{\prime}}{\Gamma(\alpha)} \sum_{s=1-\alpha}^{t-\alpha}(t-s-1)^{(\alpha-1)}(s+\alpha)^{(\eta)}\left((s+\alpha)^{(-\gamma)}\right)^{q} \\
& \leq \frac{C_{f}^{\prime} \Gamma(1+\gamma q)}{\Gamma^{q}(1+\gamma) \Gamma(\alpha)} \sum_{s=1-\alpha}^{t-\alpha}(t-s-1)^{(\alpha-1)}(s+\alpha)^{(\eta)}(s+\alpha)^{(-\gamma q)} \\
& \leq \frac{C_{f}^{\prime} \Gamma(1+\gamma q)}{\Gamma^{q}(1+\gamma) \Gamma(\alpha)} \sum_{s=1-\alpha}^{t-\alpha}(t-s-1)^{(\alpha-1)}(s+\alpha)^{(\eta-\gamma q)} \\
& =\frac{C_{f}^{\prime} \Gamma(1+\gamma q)}{\Gamma^{q}(1+\gamma)} \frac{\Gamma(1+\eta-\gamma q)}{\Gamma(1+\alpha+\eta-\gamma q)}(t+\alpha)^{(\alpha+\eta-\gamma q)} \\
& \leq \frac{C_{f}^{\prime} \Gamma(1+\gamma q)}{\Gamma^{q}(1+\gamma)} \frac{\Gamma(1+\eta-\gamma q)}{\Gamma(1+\alpha+\eta-\gamma q)} t^{(\alpha+\eta+\gamma(1-q))} t^{(-\gamma)} \\
& \leq \frac{C_{f}^{\prime} \Gamma(1+\gamma q)}{\Gamma^{q}(1+\gamma)} \frac{\Gamma(1+\eta-\gamma q)}{\Gamma(1+\alpha+\eta-\gamma q)} \frac{1}{\Gamma(2-\alpha-\eta-\gamma(1-q))} t^{(-\gamma)} \\
& \leq t^{(-\gamma)}
\end{aligned}
$$

This shows that $P S \subset S$. 
The remaining proof concerning continuity of $P$ is similar to that of Lemma 3.2. Consequently, $P S$ is compact by Lemma 3.3 and hence there exists at least one solution for the problem (1.1) by Theorem 3.1.

Corollary 3.1 Assume that $\eta=0$ in $(\mathrm{H} 3)$ and that

$(\mathrm{H} 3)^{\prime}$ there exist $C_{f}^{\prime}>0, q>1$, and $\gamma \in\left(\frac{\alpha}{q-1}, \frac{1}{q}\right)$ such that

$$
|f(t, u)| \leq C_{f}^{\prime}|u(t)|^{q}
$$

Then the problem (1.1) has at least one solution $u \in S$ and the set of solutions of (1.1) is bounded in S provided with (3.11) holds.

Example 3.2 Consider the following fractional difference equations:

$$
\left\{\begin{array}{l}
{ }^{C} \Delta^{0.2} u(t)=0.5 u^{2}(t+0.2), \quad t \in \mathbb{N}_{0.8} \\
u(0)=0
\end{array}\right.
$$

where $f(t, u)=0.5 u^{2}(t)$ for $t \in \mathbb{N}_{0}$.

Since $|f(t, u)| \leq 0.5|u(t)|^{2}$, the condition (H3) holds for $\gamma=0.25$. In consequence, there exists at least one solution for the given problem in $S$ by Corollary 3.1.

Remark 3.1 Replacing the condition $q>1$ of (H3) by $0<q \leq 1$, we observe that there does not exist any solution for the problem (1.1). However, with suitable conditions on $f$, we may have existence results for the problem (1.1).

Theorem 3.4 Let $0<q \leq 1$. Assume that

(H4) there exist $C_{f}^{\prime \prime}>0, \sigma \in[1+(\alpha-1) q, 1)$ and $\gamma \in\left(0, \frac{1-\sigma}{q}\right)$, such that

$$
\frac{C_{f}^{\prime \prime} \Gamma(1-\sigma-\gamma q)}{\Gamma(1+\alpha-\sigma-\gamma q)} \leq 1
$$

Iff satisfies

$$
|f(t, u)| \leq C_{f}^{\prime \prime}(t+1)^{(-\sigma)}|u(t)|^{q}
$$

then the problem (1.1) has at least one solution $u \in S$ and the set of solutions of (1.1) is bounded in $S$.

Proof By definition of $P$, the non-increasing characteristic of $t^{(-\alpha)}$ for any $t \in \mathbb{N}$, together with (H4), Lemma 2.4 and Lemma 2.5 , for any $u \in S$, we have

$$
\begin{aligned}
\left|(P u)(t)-u_{0}\right| & \leq \frac{1}{\Gamma(\alpha)} \sum_{s=1-\alpha}^{t-\alpha}(t-s-1)^{(\alpha-1)}|f(s+\alpha, u(s+\alpha))| \\
& \leq \frac{C_{f}^{\prime \prime}}{\Gamma(\alpha)} \sum_{s=1-\alpha}^{t-\alpha}(t-s-1)^{(\alpha-1)}(s+\alpha+1)^{(-\sigma)}|u(s+\alpha)|^{q}
\end{aligned}
$$




$$
\begin{aligned}
& \leq \frac{C_{f}^{\prime \prime}}{\Gamma(\alpha)} \sum_{s=1-\alpha}^{t-\alpha}(t-s-1)^{(\alpha-1)}(s+\alpha+\gamma q)^{(-\sigma)}\left((s+\alpha)^{(-\gamma)}\right)^{q} \\
& \leq \frac{C_{f}^{\prime \prime}}{\Gamma(\alpha)} \sum_{s=1-\alpha}^{t-\alpha}(t-s-1)^{(\alpha-1)}(s+\alpha+\gamma q)^{(-\sigma)}(s+\alpha)^{(-\gamma q)} \\
& =\frac{C_{f}^{\prime \prime}}{\Gamma(\alpha)} \sum_{s=1-\alpha}^{t-\alpha}(t-s-1)^{(\alpha-1)}(s+\alpha)^{(-\sigma-\gamma q)} \\
& =\frac{C_{f}^{\prime \prime} \Gamma(1-\sigma-\gamma q)}{\Gamma(1+\alpha-\sigma-\gamma q)}(t+\alpha)^{(\alpha-\sigma-\gamma q)} \\
& \leq \frac{C_{f}^{\prime \prime} \Gamma(1-\sigma-\gamma q)}{\Gamma(1+\alpha-\sigma-\gamma q)} t^{(\alpha-\sigma+\gamma(1-q))} t^{(-\gamma)} \\
& \leq \frac{C_{f}^{\prime \prime} \Gamma(1-\sigma-\gamma q)}{\Gamma(1+\alpha-\sigma-\gamma q)} \frac{1}{\Gamma(2-\alpha+\sigma-\gamma(1-q))} t^{(-\gamma)} \\
& \leq t^{(-\gamma)}
\end{aligned}
$$

then $P S \subset S$. The remaining proof (concerning the continuity of $P$ ) is similar to that of Lemma 3.2. Therefore PS is compact by Lemma 3.3 and hence there exists at least one solution for the problem (1.1) by Theorem 3.1.

Similarly, we can have another existence result for the problem (1.1) by changing the condition on $\sigma$ in Theorem 3.4.

Theorem 3.5 Let $0<q<1$. Assume that

$(\mathrm{H} 4)^{\prime}$ there exist $C_{f}^{\prime \prime}>0, \sigma \in(\alpha, 1+(\alpha-1) q)$ and $\gamma \in\left(0, \frac{\sigma-\alpha}{1-q}\right)$, such that

$$
|f(t, u)| \leq C_{f}^{\prime \prime}(t+1)^{(-\sigma)}|u(t)|^{q}
$$

Then the problem (1.1) has at least one solution $u \in S$ and the set of solutions of (1.1) is bounded in S provided with (3.12).

Example 3.3 Consider the following fractional difference equations:

$$
\left\{\begin{array}{l}
{ }^{C} \Delta^{0.4} u(t)=0.15(t+1.4)^{(-0.8)}|\sin (u(t+0.4))|^{0.5}, \quad t \in \mathbb{N}_{0.6}, \\
u(0)=u_{0},
\end{array}\right.
$$

where $f(t, u)=0.15(t+1)^{(-0.8)}|\sin (u(t))|^{0.5}$ for $t \in \mathbb{N}_{0}$.

Since $|f(t, u)|=0.15(t+1)^{(-0.8)}|\sin (u(t))|^{0.5} \leq 0.15(t+1)^{(-0.8)}|u(t)|^{0.5}$, the condition (H4) holds for $\gamma=0.2$. Thus there exists at least one solution for the given problem in $S$ by Theorem 3.4.

\section{Acknowledgements}

The work was supported by the National Natural Science Foundation of China (No. 11671339) and Hunan Provincial Innovation Foundation For Postgraduate (CX2018B072). 
Authors' contributions

Each of the authors, JWH, LZ, YZ and BA, contributed equally to each part of this work. All authors read and approved the final manuscript.

\section{Publisher's Note}

Springer Nature remains neutral with regard to jurisdictional claims in published maps and institutional affiliations.

Received:29 December 2017 Accepted: 20 April 2018 Published online: 27 April 2018

\section{References}

1. Kilbas, A.A., Srivastava, H.M., Trujillo, J.J.: Theory and Applications of Fractional Differential Equations. North-Holland Mathematics Studies, vol. 204. Elsevier, Amsterdam (2006)

2. Diethelm, K.: The Analysis of Fractional Differential Equations. Lecture Notes in Mathematics. Springer, Berlin (2010)

3. Zhou, Y.: Basic Theory of Fractional Differential Equations. World Scientific, Singapore (2014)

4. Zhou, Y.: Fractional Evolution Equations and Inclusions: Analysis and Control. Academic Press, San Diego (2016)

5. Chang, Y.K., Kavitha, V., Arjunan, M.M.: Existence and uniqueness of mild solutions to a semilinear integrodifferential equation of fractional order. Nonlinear Anal. TMA 71(11), 5551-5559 (2009)

6. Li, Y.N., Sun, H.R., Feng, Z.: Fractional abstract Cauchy problem with order $\alpha \in(1,2)$. Dyn. Partial Differ. Equ. 13(2), 155-177 (2016)

7. Wang, J.R., Feckan, M., Zhou, Y.: A survey on impulsive fractional differential equations. Fract. Calc. Appl. Anal. 19, 806-831 (2016)

8. Zhou, Y., Peng, L.: On the time-fractional Navier-Stokes equations. Comput. Math. Appl. 73(6), 874-891 (2017)

9. Zhou, Y., Peng, L.: Weak solution of the time-fractional Navier-Stokes equations and optimal control. Comput. Math. Appl. 73(6), 1016-1027 (2017)

10. Zhou, Y., Zhang, L.: Existence and multiplicity results of homoclinic solutions for fractional Hamiltonian systems. Comput. Math. Appl. 73(6), 1325-1345 (2017)

11. Zhou, Y., Vijayakumar, V., Murugesu, R.: Controllability for fractional evolution inclusions without compactness. Evol. Equ. Control Theory 4(1), 507-524 (2017)

12. Zhou, Y., Ahmad, B., Alsaedi, A.: Existence of nonoscillatory solutions for fractional neutral differential equations. Appl. Math. Lett. 72, 70-74 (2017)

13. Atici, F.M., Eloe, P.W.: Initial value problems in discrete frational calculus. Proc. Am. Math. Soc. 137(3), 981-989 (2009)

14. Miller, K.S., Ross, B.: Fractional difference calculus. In: Univalent Functions, Fractional Calculus and Their Applications (Kōriyama, 1988). Ellis Horwood Ser. Math. Appl., pp. 139-152. Horwood, Chichester (1989)

15. Holm, M.T.: The Laplace transform in discrete fractional calculus. Comput. Math. Appl. 62(3), 1591-1601 (2011)

16. Mohan, J.J.: Analysis of nonlinear fractional nabla difference equations. Int. J. Anal. Appl. 7(1), 79-95 (2015)

17. Boulares, H., Ardjouni, A., Laskri, Y.: Existence and uniqueness of solutions for nonlinear fractional nabla difference systems with initial conditions. Fract. Differ. Calc. 7(2), 247-263 (2017)

18. Gray, H.L., Zhang, N.F.: On a new definition of the fractional difference. Math. Comput. 50(182), 513-529 (1988)

19. Atici, F.M., Eloe, P.W.: A transform method in discrete fractional calculus. Int. J. Difference Equ. 2(2), 165-176 (2007)

20. Abdeljawad, T.: On Riemann and Caputo fractional differences. Comput. Math. Appl. 62, 1602-1611 (2011)

21. Goodrich, C.S.: Existence of a positive solution to a system of discrete fractional boundary value problems. Appl. Math. Comput. 217(9), 4740-4753 (2011)

22. Chen, F.L., Liu, Z.G.: Asymptotic stability results for nonlinear fractional difference equations. J. Appl. Math. 2012 Article ID 879657 (2012)

23. Chen, F.L.: Fixed points and asymptotic stability of nonlinear fractional difference equations. Electron. J. Qual. Theory Differ. Equ. 2011, Article ID 39 (2011)

24. Chen, F., Luo, X., Zhou, Y.: Existence results for nonlinear fractional difference equation. Adv. Differ. Equ. 2011, Article ID 713201 (2011)

25. Isaia, F.: On a nonlinear integral equation without compactness. Acta Math. Univ. Comen. 75, 233-240 (2006)

\section{Submit your manuscript to a SpringerOpen ${ }^{\circ}$ journal and benefit from:}

- Convenient online submission

- Rigorous peer review

- Open access: articles freely available online

- High visibility within the field

- Retaining the copyright to your article

Submit your next manuscript at $>$ springeropen.com 